\title{
Using Social Media to Engage Communities with Research: Basics ${ }^{1}$
}

\author{
Kathryn A. Stofer, Lisa Lundgren, Kirsten Hecht, and Tyus D. Williams ${ }^{2}$
}

\section{Introduction}

This publication defines social media, presents reasons for using it to share science, and identifies multiple platforms available for use now. We provide an introduction to and overview for a series on getting the most out of social media for sharing science or STEM, including agricultural research. Our primary goal is to assist people working through social media to broaden the community engaged with research. The series includes additional publications on social media planning, evaluating social media engagement, and social media for branding, among other topics. For an overview of all the documents in this series, please see the Appendix.

\section{Social Media as Engagement}

Connecting with audiences via social media is one form of a broader suite of "public engagement" strategies, also sometimes thought of as informal education, science communication, or science outreach, all of which are under the purview of cooperative extension activities as well. For more on the distinction in these terms, see EDIS document AEC610, Getting Engaged: "Public" Engagement Practices for Researchers (https://edis.ifas.ufl.edu/wc272), and the related EDIS series on public engagement. "Social media engagement" specifically is a quantitative measure reported to each social media account administrator. It is an amalgamation of the number of potential post viewers and the clicks, shares, comments, retweets, likes, or other actions on each post, or essentially, a rate of reach divided by users (Bugeaud et al., 2016). For more information, please see the document in this series on social media evaluation (forthcoming).

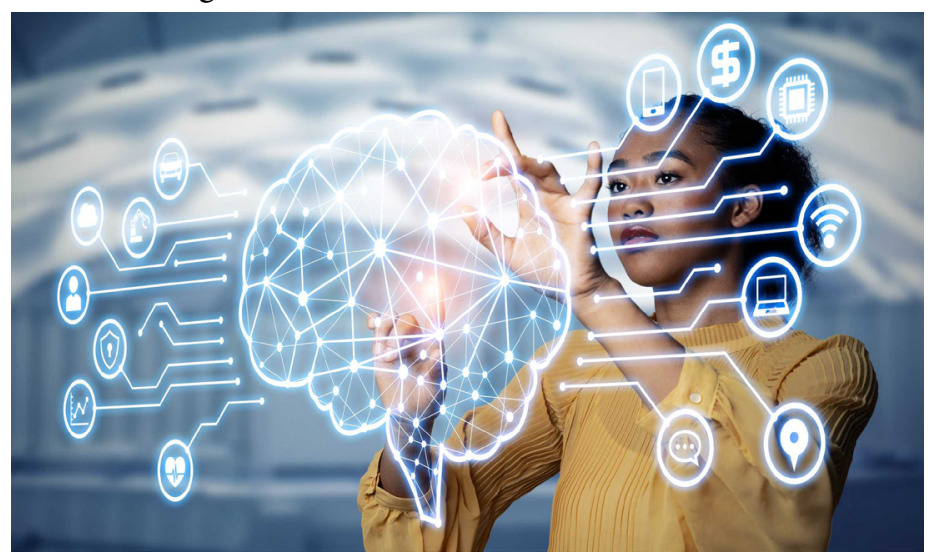

Credits: Gettyimages.com

\section{Why use social media?}

"The Internet has paved the way for building relationships through the exchange of information in a way that has never been experienced before" (Fernandez and Rumble, 2012, "Why Use Social Media?"). Social media allows multidirectional interaction through the use of words, pictures, videos, and audio (shown in Figure 2), appealing to more senses and going beyond traditional one-way broadcast modes. Social media adds value to information

1. This document is AEC698, one of a series of the Department of Agricultural Education and Communication, UF/IFAS Extension. Original publication date November 2020. Visit the EDIS website at https://edis.ifas.ufl.edu for the currently supported version of this publication.

2. Kathryn A. Stofer, research assistant professor, Department of Agricultural Education and Communication, UF/IFAS Extension; Lisa Lundgren, postdoctoral research associate, Neag School of Education, University of Connecticut; Kirsten Hecht, PhD candidate, interdisciplinary ecology, University of Florida; and Tyus D. Williams, community field ecology technician, University of Nevada-Reno; UF/IFAS Extension, Gainesville, FL 32611.

The Institute of Food and Agricultural Sciences (IFAS) is an Equal Opportunity Institution authorized to provide research, educational information and other services

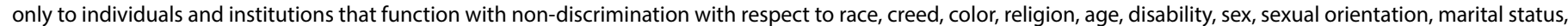

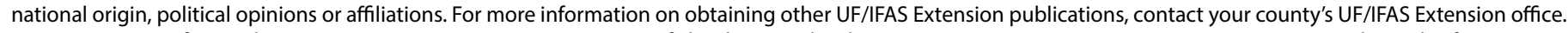
U.S. Department of Agriculture, UF/IFAS Extension Service, University of Florida, IFAS, Florida A \& M University Cooperative Extension Program, and Boards of County Commissioners Cooperating. Nick T. Place, dean for UF/IFAS Extension. 
being shared among researchers and nonresearcher audiences. With fewer gatekeepers than traditional media or even organizational public affairs offices, social media can also support transparency and authenticity.

Social media presents a complementary platform to traditional media for sharing the latest news from science and STEM research, including agriculture. Social media can be more akin to word of mouth or diffusion of information through trusted communities than traditional media. It also generally offers a more interactive communication format than traditional media, even newspapers and magazines that publish online and provide comment sections. Social media is a top source of news for US adults as of late 2019 (Schaeffer, 2019), including science news, which is an important medium for younger adults in particular to learn about science (Funk, Gottfried, \& Mitchell, 2019).

For researchers and STEM professionals, two primary, though not exhaustive, reasons to use social media are: 1) academic reasons directly related to improving your work and 2) science communication or science engagement reasons to share your work with broader audiences (Collins, Shiffman, \& Rock, 2016). Of course, there may be other reasons to take up social media, but we will focus on these motivations to focus our recommendations in this series. Academically, social media can facilitate networking, increase the visibility of your publications and work in general, and assist with keeping up with news and publications in your field and related fields. For example, conferences use hashtags to connect researchers together when it is impossible to attend every session (Bombaci et al., 2015). In addition, for some types of work, social media can allow you to harness the power of crowdsourcing or citizen science and collect or analyze data with the assistance of a vast array of participants. Social media can also be a way to gather data on your audience to inform your work, get feedback on your ideas as they are in progress, or find inspiration for new directions for your work.

To meet science communication or public engagement goals, sharing on social media can help spread factual information and knowledge, specifically to counteract alternative conceptions or release new findings. For example, researchers reported that automated Twitter bots spread misinformation about cannabis's health benefits in 2018 (Allem \& Ferrara, 2018); researchers can help counteract this spread by pointing users to reputable data. Beyond simply sharing knowledge, social media could have a stronger influence on behavior change by improving attitudes about science-for instance, by portraying more scientists as regular humans with hobbies and families and allowing more people to interact with them outside traditional academic venues. Getting to know scientists through social media also can help build trust in science as an institution and raise the profiles of scientists who are from underrepresented backgrounds, such as those with disabilities and those from diverse ethnic, gender, and socioeconomic backgrounds.

Only about $5 \%$ of Americans work in STEM ${ }^{1}$ today (Stofer, 2019). For agriculture in particular, the number of Americans with ties to food and fiber production has fallen from $30 \%-50 \%$ in the mid-1900s to less than $1 \%$ listing production agriculture as their occupation, and 2\% directly involved in agriculture full time (Baker \& Irani, 2012). Engaging through social media can share your work with a broad audience, build trust, and inspire people to consider STEM careers. By adopting social media as part of public engagement strategies, researchers and scientists amplify their voice.

${ }^{1}$ Throughout this series, we will use "science" and "STEM" interchangeably to refer to any of a number of disciplines with a tradition of rigorous research and iterative development with peer feedback, including agriculture, technology, engineering, math, medicine, and others.

\section{Getting Started with Social Media}

To get started on social media, a first step should be to choose a platform, that is, the site where users post messages and files and react to each other's posts (Telg \& Irani, 2012). The platform you choose should align with your objectives and goals, primarily reaching the right audience and providing the discussion and sharing tools to interact with them.

Whatever you choose, consistency in one platform is better than several inactive accounts on multiple platforms, that is, depth is better than breadth in most cases. Make sure you have one platform fully integrated in your or your lab's workflow before taking on others; the exception to this could be purely sharing information without interaction, which can be accomplished on multiple platforms through management tools discussed in EDIS publication AEC699, SMART Social Media: Planning for Success (https://edis.ifas. ufl.edu/wc362), the second in this series. Once you have mastered one platform, you may find it easy and desirable to repackage content, such as videos or images, and share them on multiple platforms to reach different audiences. 
General tips:

- Be sure to check with your organization as to their social media policies. They may also offer professional development on how to use social media, tips for building an audience, suggestions for use in your organization, or assistance on setting up an account to represent yourself or your part of the organization.

- UF/IFAS social media guidelines and assistance (https://ics.ifas.ufl.edu/our-services/social-media/) can be found through UF/IFAS Communications, and UF's policies are found through Human Resources.

- Regardless of platform, you will need to build a community. This may be through "friends," where you must mutually agree to connect and then have access to each other's content (for example, Facebook personal accounts, LinkedIn) or "followers," where someone chooses to follow content you share, but you don't necessarily have to view their content (Twitter, Instagram) or may not be allowed to view their content without requesting to follow them in return.

- Virtually every platform will offer suggestions of people to connect with. You may choose to have people request to follow you or allow anyone to follow you without your approval.

- Consider each platform's "traditions" for building an audience; for example, Twitter users may often "follow back" people who follow them.

- Connecting with important organizations in your field and commenting on their work is another way to build your audience and visibility for your work, because the organization's connections then may connect with you as well.

- For additional suggestions on building a community and identifying the types of people to connect with, see the EDIS publications AEC699,SMART Social Media: Planning for Success (https://edis.ifas.ufl.edu/ wc362), and Building Your Brand through Best Practices (forthcoming) in this series.

- Each platform has different ways to connect with people and share content. For example, each individual Facebook post can be shared with everyone on Facebook, only a group you have designated, or only yourself. However, on Twitter, each post is open to anyone on Twitter through searching by topic or by hashtag (discussed below), but a post may not appear in users' accounts unless they follow you.

- These platforms may change these settings and their general offerings frequently and somewhat unexpectedly.
Be sure to keep this in mind, and be aware that your strategy might need to change over time.

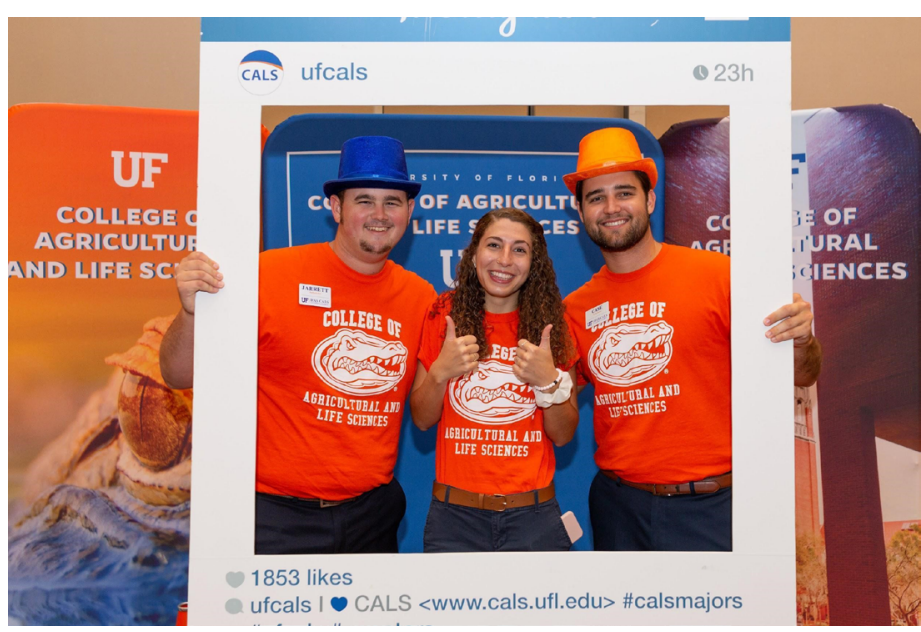

Figure 2. Example of a social media post opportunity: students posing with a photo frame.

Credits: UF/IFAS

Next, we'll discuss the most popular platforms' characteristics and current audiences. However, features change regularly, and we recommend investigating the platform you wish to use before committing to it. Some platforms are banned by certain countries, for example.

- Facebook: Facebook may be currently the most versatile platform with the broadest community in terms of age, interest, and location.

- Several types of tools:

- Individual accounts linked to a person

- Can keep a personal profile private and share your research professionally, even creating a group for your research lab.

- "Pages" for organizations, such as a research lab, where multiple people could contribute

- Groups that may be led by a few people but are open to many Facebook users and organize primarily around common interests. Examples include the Parkinson's Research Interest group, which brings together researchers and patients but is not a support group, or Snake Identification, where researchers and interested people share and discuss snakes.

- You can be active on the platform by posting updates about your day-to-day research activities, sharing messages and images about your work or your broader discipline, promoting career opportunities, and interacting with organizations or friends.

- You will also be able to see content your connections interact with, including your own posts and items shared with you. 
- All parties can be involved in sharing information and actively participate in multiway communication.

- Twitter

- Tweets (Twitter posts) are public by default.

- Users can set up lists of accounts as subgroups of accounts they are following to help curate their feeds by interest.

- Hashtags and @'s (mentions) can bring new people into a conversation or invite particular users to the conversation. Alternatively, searching for other tweets with particular hashtags can help you become part of a larger conversation.

- A few popular hashtags are:

- \#ActualLivingScientist, where scientists introduce themselves and their work

- \#DisabledAndSTEM and similar for conversations about accessibility and diversity

- \#SciEngage for conversations about public engagement with science

- \#FieldworkFail, sharing less-than-perfect but often hilarious real life adventures.

- Blogs provide a place for long-form posts that are often essay-style, allowing perhaps for expanded discussion once interest is piqued in another platform.

- Your blog can also link back to your other social media.

- You can also set up pages of resources such as links to popular journals in your field or public engagement resources.

- Posting links to these pages on a regular basis on other platforms or linking them in your biographies on these platforms can also drive traffic and build your reputation in the field.

- Be sure to optimize your keywords for your blog to promote its position in search engines.

- UF/IFAS Communications provides faculty and staff blogging tools through WordPress.

- Video-sharing sites include YouTube (now owned by Google) and Vimeo.

- Users can create playlists of items and follow other users, but after each video, an autoplay option may start an unrelated video.

- Some internet personalities produce full "episodes" on an ongoing topic of interest, often with video editing and even soundtracks and other production capabilities. However, for scientists, the best use may be as a permanent storage site for linking to videos that you may promote using links on other sites, given limited capabilities for feeds and keywords.

- YouTube/Vimeo can be a good place to put videos produced for other purposes, such as clips of traditional media appearances or presentations for conferences.

- YouTube also offers automatic captioning for recorded items, helpful for audiences with hearing disabilities. Be sure to review and correct the autocaptioning as needed.

- To see a discussion of YouTube vs. Vimeo, check out this LifeWire blog post from March 2020.

- LinkedIn is used widely in the corporate world, primarily for career networking.

- Users must request connections, and profiles often list education, experience, and other résumé-related information.

- Users can post articles similar to blog posts to an audience interested in career- or workplace-relevant information.

- TikTok is primarily used for the creation of short videos (the average length is 15 seconds) that often feature music in the background.

- Users can follow one another or view the content of users who are popular on the platform.

- Users who are interested in STEM post content related to their day-to-day life (e.g., what it is like to work in a research lab).

- Often, the content features the Gen Z brand of humor: absurdist and nihilistic (Mercado, 2019).

- Pinterest focuses more on sharing do-it-yourself ideas and recipes via images and videos. It's not widely used for science as far as we know, but you could link these to other accounts to share images.

- New platforms may emerge over time, particularly catering to other audiences. Joining these platforms as an early adopter may make sense if your audience joins them also. Otherwise, there may be a trade-off in how you spend your engagement resources, because some platforms may not catch on with a wide audience or may fail or disappear after a short time.

A note on DMs (direct messages): messaging individual or multiple users is also built in to most if not all of these systems, akin to texting within the platform. DMs can be a resource for contacting others privately, but users may be able to restrict these messages in some cases. We discuss DM use more in the forthcoming EDIS document on 
branding and social media, Building Your Brand through Best Practices.

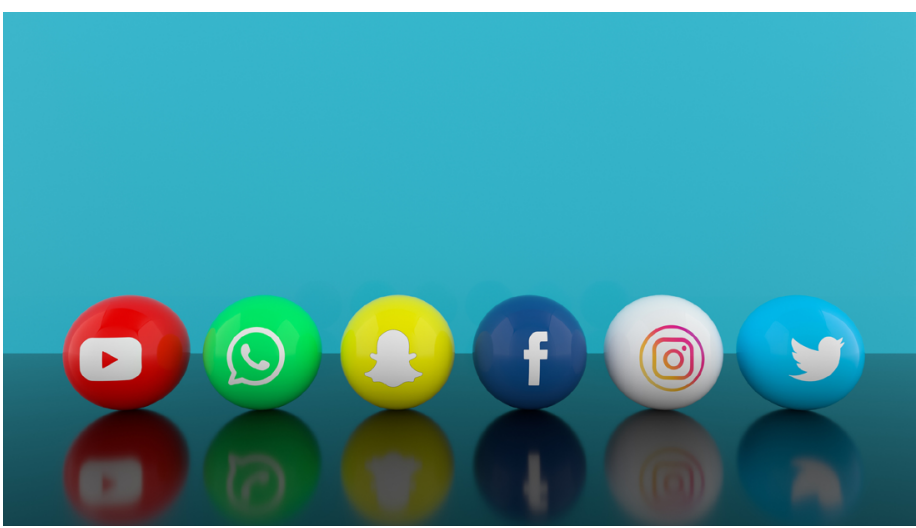

Figure 3. Various social media icons.

Credits: Serdarbayraktar/Gettyimages.com

There is no limit to how many of these platforms you can use to share your science. However, remember: quality over quantity. Having one platform that you keep up with and build a community on is more valuable than four platforms that are not active or up to date. Other publications in this series will cover creating a social media strategy that provides value on an ongoing basis to the audience or audiences with whom you wish to interact and how to evaluate your efforts to ensure you are reaching who you intend to.

\section{Summary}

Social media and its many platforms are here to stay; in fact, companies and industries of all sizes now view social media as a mandatory element of their marketing strategies (Hanna et al., 2011). UF/IFAS personnel have tools to report their social media contacts as part of their regular workload, and public engagement and outreach are growing in recognition in academic promotion and tenure packages. "Marketing" through social media for researchers can help build new collaborations and inspire future generations. Reaching beyond typical academic audiences may be a necessary part of future funding efforts or may be part of an Extension appointment. Integrating and building a social media presence for your organization or research can help you connect to other professional contacts as well as broader audiences who may both benefit from and contribute to your work. For an overview of all the documents in this series, please see the Appendix.

\section{References}

Allem, J.-P., \& Ferrara, E. (2018). Could social bots pose a threat to public health? American Journal of Public Health, 108(8), 1005-1006.
Baker, L., \& Irani, T. (2012). The impact of new media on policy affecting agriculture. Journal of Applied Communications, 98(3), 17-31.

Bombaci, S. P., Farr, C. M., Gallo, H. T., Mangan, A. M., Stinson, L. T., Kaushik, M., \& Pejchar, L. (2016). Using Twitter to communicate conservation science from a professional conference. Conservation Biology, 30(1), 216-225.

Collins, K., Shiffman, D., \& Rock, J. (2016). How are scientists using social media in the workplace? Plos One, 11(10), e0162680.

Funk, C., Gottfried, J., \& Mitchell, A. (2017, September 20). Most Americans see at least some science posts on social media but tend to distrust what they see. Pew Research Center's Journalism Project. https://www.journalism. org/2017/09/20/most-americans-see-at-least-some-scienceposts-on-social-media-but-tend-to-distrust-what-they-see/

Hanna, R., Rohm, A., \& Crittenden, V. (2011). We're all connected: The power of the social media ecosystem. Business Horizons, 54, 265-273.

Mercado, W. (2019, June 18). The shocking and absurdist humor of Gen Z. Medium.https://medium.com/@williamfmercado/ the-shocking-and-absurdist-humor-of-gen-z-f3d6e 363d42b

Moreau, E. (2020, March 3). Vimeo or YouTube: Which is better? Lifewire. https://www.lifewire.com/ vimeo-vs-youtube-which-is-better-3486370

Perrin, A., \& Anderson, M. (2019, April 10). Share of U.S. adults using social media, including Facebook, is mostly unchanged since 2018. Pew Research Center. https:// www.pewresearch.org/fact-tank/2019/04/10/share-of-u-sadults-using-social-media-including-facebook-is-mostlyunchanged-since-2018/

Schaeffer, K. (2019, December 20). U.S. has changed in key ways in the past decade, from tech use to demographics. Pew Research Center. https:// www.pewresearch.org/fact-tank/2019/12/20/ key-ways-us-changed-in-past-decade/

Telg, R., \& Irani, T. (2012). Agricultural communications in action: A hands-on approach (1st ed.). Clifton Park, NY: Delmar, CENGAGE Learning. 
Wojcik, S., \& Hughes, A. (2019, April 24). Sizing up Twitter users. Pew Research Center: Internet, Science \& Tech. https://www.pewresearch.org/internet/2019/04/24/ sizing-up-twitter-users/

\section{Acknowledgments}

This document is inspired by and based on an earlier archived document, AEC558, Getting the Most out of Social Media: What Is Social Media? by Jessica C. Fernandez and Joy N. Rumble.

\section{Appendix: Documents in This}

\section{Series}

Using Social Media to Engage Communities with Research: Basics (this document)

An overview of uses of social media for researchers, platforms, and engagement types.

Using Social Media to Engage Communities with Research: Social Media Planning

How to create a plan to determine your social media strategy including goal-setting, resource mobilization, and platform choice.

Using Social Media to Engage Communities with Research: Evaluating Your Success

How to determine progress toward your goals using platform metrics and qualitative techniques.

Using Social Media to Engage Communities with Research: Creating a Community through Best Practices

How to find your voice and build a community on social media.

Using Social Media to Engage Communities with Research: Diversity and Inclusion

How to use social media to promote marginalized voices and encourage a wider range of voices in conversations around research.

Using Social Media to Engage Communities with Research: How to Share Complex Research

How to break down a research paper into a series of engaging and concise social media posts.
Using Social Media to Engage Communities with Research: Social Network Analysis for Community Evaluation

How to determine who your social media community is by analyzing followers and connections. 
Table 1. Feature comparison of several popular social media platforms.

\begin{tabular}{|c|c|c|c|c|c|}
\hline & Facebook & Twitter & Instagram & Blogs & YouTube/Vimeo \\
\hline Global users ${ }^{2}$ & 1 billion+ & 100 million+ & 100 million+ & $\mathrm{N} / \mathrm{A}$ & $\begin{array}{l}2 \text { billion+ monthly } / 2 \\
\text { million monthly }\end{array}$ \\
\hline Audience & $\begin{array}{l}\text { Broadest, mix of public } \\
\text { entities and private } \\
\text { connections }\end{array}$ & $\begin{array}{l}\text { Young, highly } \\
\text { educated, mix of public } \\
\text { entities and private } \\
\text { users }^{3}\end{array}$ & $\begin{array}{l}\text { Younger, primarily } \\
\text { private connections } \\
\text { but some public } \\
\text { accounts }\end{array}$ & $\begin{array}{l}\text { Specialized followers of } \\
\text { individual bloggers }\end{array}$ & $\begin{array}{l}\text { Widely used by adults } \\
\text { in the United States }\end{array}$ \\
\hline Format & $\begin{array}{l}\text { Textual posts of any } \\
\text { length with options to } \\
\text { add video, audio, URL } \\
\text { links, and images }\end{array}$ & $\begin{array}{l}\text { 280-character "tweets" } \\
\text { with options to add } \\
\text { video, audio, URL links, } \\
\text { and images }\end{array}$ & $\begin{array}{l}\text { Images and videos } \\
\text { primarily, with short } \\
\text { captions }\end{array}$ & $\begin{array}{l}\text { Essay-style posts of } \\
\text { irregular length and } \\
\text { frequency }\end{array}$ & Videos \\
\hline Tools & $\begin{array}{l}\text { Individual accounts } \\
\text { Organization pages } \\
\text { Interest groups }\end{array}$ & $\begin{array}{l}\text { Individual accounts, } \\
\text { though organization/ } \\
\text { group accounts and } \\
\text { "takeovers" by guests } \\
\text { are common }\end{array}$ & Individual accounts & $\begin{array}{l}\text { Multiple platforms, } \\
\text { such as through } \\
\text { employer, or build- } \\
\text { your-own web page, or } \\
\text { Linkedln }\end{array}$ & Individual accounts \\
\hline Features & $\begin{array}{l}\text { Can tag posts with } \\
\text { other users to whom } \\
\text { you are connected } \\
\text { Live video broadcast } \\
\text { capability }\end{array}$ & $\begin{array}{l}\text { Hashtags allow for } \\
\text { following topics of } \\
\text { interest across users } \\
\text { @ mentions can call } \\
\text { attention to particular } \\
\text { users and thread } \\
\text { conversations } \\
\text { Retweets of posts may } \\
\text { amplify visibility }\end{array}$ & $\begin{array}{l}\text { Hashtags in captions } \\
\text { and comments } \\
\text { Live video broadcast } \\
\text { capability }\end{array}$ & $\begin{array}{l}\text { Completely } \\
\text { customizable }\end{array}$ & $\begin{array}{l}\text { Both are often available } \\
\text { as an app on TV } \\
\text { streaming systems } \\
\text { Both have } \\
\text { monetization/tip } \\
\text { options }\end{array}$ \\
\hline \multicolumn{6}{|c|}{$\begin{array}{l}\text { 2 Source: Perrin \& Anderson (2019) for all numbers except Vimeo (Moreau 2020) } \\
{ }^{3} \text { Wojcik \& Hughes (2019) } \\
{ }^{4} \text { Individual accounts may be "managed" by an organization who allows multiple users to post on their behalf. Check Terms of Service for each } \\
\text { platform for any restrictions. }\end{array}$} \\
\hline
\end{tabular}

